ARTICLE

Received 19 Dec 2013 | Accepted 14 May 2014 | Published 17 Jun 2014

DOl: $10.1038 /$ ncomms5128

OPEN

\title{
Encapsulation kinetics and dynamics of carbon monoxide in clathrate hydrate
}

\author{
Jinlong Zhu ${ }^{1,2,3}$, Shiyu Du ${ }^{4,5}$, Xiaohui $\mathrm{Yu}^{1,2}$, Jianzhong Zhang ${ }^{1}$, Hongwu $\mathrm{Xu}^{6}$, Sven C. Vogel ${ }^{1}$, \\ Timothy C. Germann ${ }^{4}$, Joseph S. Francisco ${ }^{7}$, Fujio Izumi ${ }^{8}$, Koichi Momma ${ }^{8, \dagger}$, Yukihiko Kawamura ${ }^{8, \dagger}$, \\ Changqing $\operatorname{Jin}^{2} \&$ Yusheng Zhao $1,2,3$
}

Carbon monoxide clathrate hydrate is a potentially important constituent in the solar system. In contrast to the well-established relation between the size of gaseous molecule and hydrate structure, previous work showed that carbon monoxide molecules preferentially form structure-I rather than structure-II gas hydrate. Resolving this discrepancy is fundamentally important to understanding clathrate formation, structure stabilization and the role the dipole moment/molecular polarizability plays in these processes. Here we report the synthesis of structure-II carbon monoxide hydrate under moderate high-pressure/low-temperature conditions. We demonstrate that the relative stability between structure-I and structure-II hydrates is primarily determined by kinetically controlled cage filling and associated binding energies. Within hexakaidecahedral cage, molecular dynamic simulations of density distributions reveal eight low-energy wells forming a cubic geometry in favour of the occupancy of carbon monoxide molecules, suggesting that the carbon monoxide-water and carbon monoxide-carbon monoxide interactions with adjacent cages provide a significant source of stability for the structure-II clathrate framework.

\footnotetext{
${ }^{1}$ LANSCE, Los Alamos National Laboratory, Los Alamos, New Mexico 87545, USA. ${ }^{2}$ National Laboratory for Condensed Matter Physics, Institute of Physics, Chinese Academy of Sciences, Beijing 100190, China. ${ }^{3}$ HiPSEC, Department of Physics and Astronomy, University of Nevada, Las Vegas, Nevada 89154 USA. ${ }^{4}$ T-Division, Los Alamos National Laboratory, Los Alamos, New Mexico 87545, USA. ${ }^{5}$ Division of Functional Materials and Nanodevices, Ningbo Institute of Materials Technology and Engineering, Chinese Academy of Sciences, Ningbo, Zhejiang 315201, China. ${ }^{6}$ EES Division, Los Alamos National Laboratory, Los Alamos, New Mexico 87545, USA. ${ }^{7}$ Departments of Chemistry and Earth and Atmospheric Science, Purdue University, West Lafayette, Indiana 47906, USA. ${ }^{8}$ National Institute for Materials Science, 1-1 Namiki, Tsukuba, Ibaraki 305-0044, Japan. ${ }^{\dagger}$ Present addresses: National Museum of Nature and Science, 4-1-1 Amakubo, Tsukuba, Ibaraki 305-0005, Japan (K.M.); Research Center for Neutron Science and Technology, Comprehensive Research Organization for Science and Society, 162-1 Shirane Shirakata, Tokai-mura, Naka-gun, Ibaraki 319-1106, Japan (Y.K.). Correspondence and requests for materials should be addressed to X.Y. (email: yuxh@iphy.ac.cn) or to H.X. (email: hxu@lanl.gov) or to Y.Z. (email: Yusheng.Zhao@unlv.edu).
} 
C lathrate hydrates are non-stoichiometric compounds consisting of various types of hydrogen-bonded water polyhedral cages containing guest molecules ${ }^{1}$. Depending on the cage geometry and connectivity, hydrates usually crystallize in one of the two most common structures, structure-I (sI, space group $P m \overline{3} n$ ) and structure-II (sII, $F d \overline{3} \mathrm{~m}$ ), both of which are cubic. The sI unit cell contains two pentagonal dodecahedra $\left(5^{12}\right)$ cages and six tetradecahedron $\left(5^{12} 6^{2}\right)$ cages. In a sII unit cell, there are $165^{12}$ cages and 8 large hexakaidecahedral $5^{12} 6^{4}$ cages. The structure formed largely depends on the size of guest molecules enclosed in the cages. $\mathrm{sI}$ is often formed with small guest molecules such as methane and carbon dioxide, while sII can enclose larger guests such as propane and isobutene. Interestingly, very small molecules, such as $\mathrm{H}_{2}, \mathrm{~N}_{2}$ and $\mathrm{O}_{2}$, also form sII hydrates. These molecules can stabilize the ice-like framework because sII has a larger number of small cages and because the large $5^{12} 6^{4}$ cages can accommodate multiple small molecules. There is yet a third hydrate structure, known as structure $\mathrm{H}(\mathrm{sH})^{1,2}$, which crystallizes in a $P 6 / \mathrm{mmm}$ structure consisting of layers of $5^{12}$ cages alternating with those of $4^{3} 5^{6} 6^{3}$ and $5^{12} 6^{8}$ cages. The $5^{12}$ and $4^{3} 5^{6} 6^{3}$ cages are occupied by small molecules such as methane while the molecules in the large interstitial icosahedral $\left(5^{12} 6^{8}\right)$ cages are typically larger than $7 \AA$, such as neohexane and adamantane ${ }^{1,3}$. Therefore, the stabilization of the $\mathrm{sH}$ structure requires the enclosure of both small and large guest molecules. Generally speaking, multiple hydrate structures of small single gases such as $\mathrm{CO}_{2}, \mathrm{~N}_{2}, \mathrm{Xe}, \mathrm{Kr}$ and $\mathrm{CH}_{4}$ (refs 4-10) can be formed at various pressure and temperature conditions.

As a cosmochemically important gas molecule and a predominant form of carbon in solar nebulae ${ }^{11}$, carbon monoxide (CO) occurs in mixed gas hydrates $\left(\mathrm{CO}_{2}, \mathrm{H}_{2}, \mathrm{~N}_{2}\right.$ and so on), and the formation of these clathrate phases may play important roles in the formation of nebulae, comets and the outer planets of the solar system ${ }^{12,13}$. For instance, the high $\mathrm{CO} / \mathrm{N}_{2}$ ratios in primordial Titan may indicate that $\mathrm{CO}$ had a higher possibility to form clathrate relative to $\mathrm{N}_{2}$ if both gases were accreted as clathrates ${ }^{12}$. In addition, these enclosure compounds are environmentally relevant, as they have potential applications in the sequestration of greenhouse gases and separation of industrial flue gases.

For a given method of measurement, a molecule of $\mathrm{CO}$ has a similar size to those of $\mathrm{N}_{2}$ and $\mathrm{O}_{2}$; consideration of only the size effect therefore favours a sII clathrate, based on the guest-hydrate cavity size relation ${ }^{14}$. In addition, based on the theoretical calculation, Miller ${ }^{13}$ predicted that sII CO clathrate is energetically more favourable than its sI counterpart. However, contrary to this prediction, all experimental studies show that $\mathrm{CO}$ forms sI clathrate hydrate under moderate pressure and temperature conditions ${ }^{15-20}$. Thermodynamic calculations using the classical Lennard-Jones-Devonshire cell model also concluded that $\mathrm{CO}$ sI hydrate would be more stable, giving a dissociation pressure that is $20 \%$ lower than that for sII clathrate $^{12}$. Recently, Dartois ${ }^{20}$ modelled the equilibrium phase diagram of CO clathrate in $P-T$ space using the Ballard and Sloan's formalism ${ }^{21}$ and the Kihara potential for $\mathrm{CO}^{17}$. The calculated stability curve for sII lies slightly below that for sI, indicating that the sII clathrate is thermodynamically favoured.

To date, the controversy between experiments and theoretical predictions has not been resolved. One school of thought attributes the discrepancy to the electrostatic properties (that is, dipole moment and molecular polarizability) of the CO molecule during clathration. This hypothesis is worth exploring because the dipole moment is an important factor that influences the hydrate stability; other factors include the size of guest molecules, short range guest-host interactions and hydrogen bonding ${ }^{22}$. Unlike other molecules such as $\mathrm{H}_{2}, \mathrm{CO}_{2}, \mathrm{CH}_{4}$ and especially $\mathrm{N}_{2}$, which is isoelectronic with $\mathrm{CO}$, the $\mathrm{CO}$ molecule has a non-zero dipole moment of $0.122 \mathrm{D}$. The exact nature of the dipole interaction between $\mathrm{CO}$ and clathrate cages is not currently known. It has also been argued that the crystallization of $\mathrm{CO}$ clathrate requires high concentration of gaseous molecules to overcome a significant energy barrier to nucleation. As a result, nucleation would be more efficient in a pressure region where the sI CO clathrate is favoured ${ }^{20}$. In the absence of thermodynamic equilibrium data for the sII CO clathrate, such as framework stability and cage occupancy, one is left with an inconclusive hypothesis that warrants further experimental validation.

In this work, we conduct time-dependent neutron diffraction experiments on the $\mathrm{D}_{2} \mathrm{O}(\mathrm{s})-\mathrm{CO}(\mathrm{g})$ system at simultaneously low temperature and high hydrostatic pressure conditions. We successfully synthesize sII CO hydrate and determine its cage gas filling as a function of time. We also perform density functional theory (DFT) calculations to evaluate the thermodynamic properties of this system. On the basis of the obtained results, the stability of $\mathrm{CO}$ hydrates appears to be primarily controlled by the cage occupancy and the binding energy between $\mathrm{CO}$ and $\mathrm{D}_{2} \mathrm{O}$ molecules. Using combined analyses of Rietveld refinement, maximum entropy method (MEM) and molecular dynamics (MD) simulations, we investigate the distribution of $\mathrm{CO}$ molecules in sII cages and their interaction with, and stabilization in, the host water framework.

\section{Results}

Neutron diffraction. Figure 1 shows neutron diffraction patterns of CO hydrates obtained in two different synthesis experiments. Consistent with the earlier finding ${ }^{15,16}$, CO hydrate crystallized in sI at 173 bar and $243 \mathrm{~K}$. Over the initial period of two and a half weeks, the $\mathrm{CO}$ gas continued to react with $\mathrm{D}_{2} \mathrm{O}$ ice to form sI hydrate. Interestingly, in both experiments, sII hydrate started to form and coexisted with sI hydrate after staying at the same

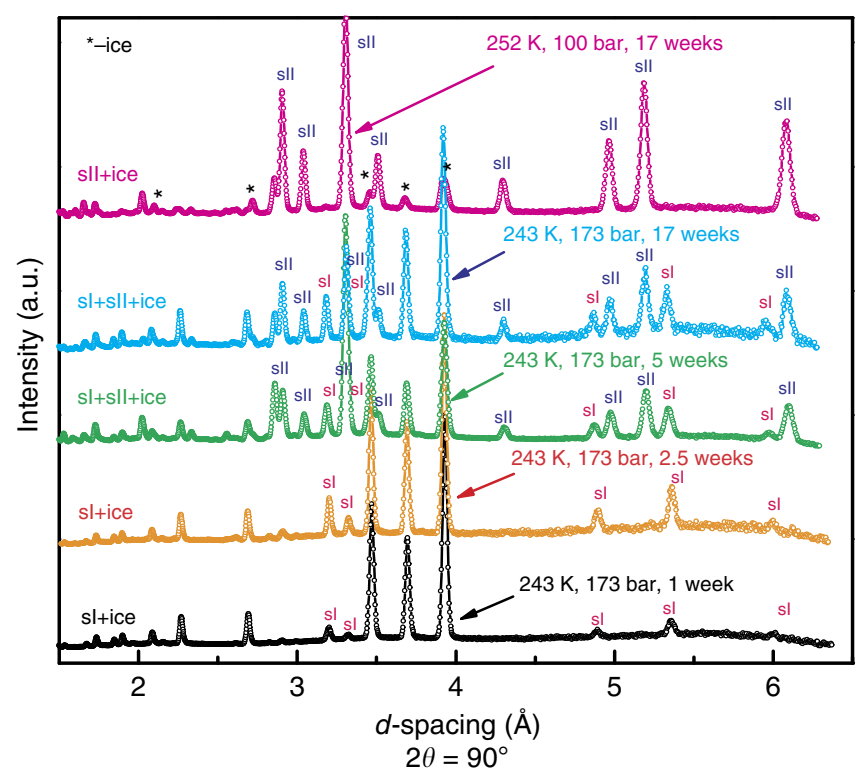

Figure 1 | Neutron diffraction data. Time-dependent neutron diffraction patterns showing carbon monoxide clathrate formation. The diffraction patterns collected at 173 bar after 1, 2.5 and 17 weeks are from one synthesis run. The patterns collected at 173 bar after 5 weeks and at 100 bar after 17 weeks are from another synthesis run. The conditions shown in the figure are the synthesis pressures and temperatures. All neutron diffraction data were obtained at $260 \mathrm{~K}$ and at indicated pressures. 
conditions for additional amounts of time. These observations indicate that the formation of sII CO hydrate at these $P-T$ conditions is thermodynamically favoured but not kinetically favoured. They also indicate that between the two competing pathways leading to $\mathrm{CO}$ hydrates, the activation energy for sI is lower than that for sII. Hence, the earlier nucleation of sI hydrate is kinetically controlled. Further inspection of Fig. 1 reveals that after the appearance of sII hydrate the relative diffraction intensities between sI and sII hydrates do not show notable changes with time, indicating that the two hydrates may have formed via two separate nucleation paths. When the pressure was decreased from 173 to 100 bar while the temperature was kept at $252 \mathrm{~K}$ for 12 weeks, a pure phase sII hydrate was finally obtained. To gain further insight into the kinetics of sII hydrate formation, we performed a third experiment at a lower pressure, $100 \mathrm{bar}$, and $252 \mathrm{~K}$. However, neither sI nor sII hydrate was observed over a period of three and a half weeks. This is not totally unexpected because the formation of both sI and sII CO hydrates requires a threshold pressure to trigger the nucleation of the clathrate phase. Clearly, in the system $\mathrm{CO}(\mathrm{g})-\mathrm{D}_{2} \mathrm{O}(\mathrm{s})$, the applied pressure of 100 bars was too low to fulfil this requirement.

In a given system, pressure and temperature are the most important thermodynamic parameters. From this point of view, it is quite unexpected that $\mathrm{D}_{2} \mathrm{O}$ ice first reacts with $\mathrm{CO}$ molecules to form sI hydrate at 173 bar and $243 \mathrm{~K}$ and then to form sII hydrate at the same $P-T$ conditions, either through the same reaction or a phase transformation. However, composition is yet another important thermodynamic variable, especially for clathrate hydrates. Because hydrates are intrinsically non-stoichiometric, their compositions are closely tied to the filling of the cages, which would in turn influence their thermodynamic stability. To gain insight into this effect, we refined the cage occupancies using the data shown in Fig. 1. Our results indicate that the small cages in sI and sII clathrates are fully or close to fully occupied ( $100 \pm 5 \%$ uncertainty); therefore, to a first approximation, they were fixed at a full occupancy during the subsequent refinements. For $5^{12} 6^{2}$ cages in sI, the occupancies are $1.13,1.18,1.34$ and 1.39 , in hydrates synthesized for $1,2.5,5$ and 17 weeks, respectively. For $5^{12} 6^{4}$ cages in sII, they are 1.59 and 2 , in hydrates synthesized for 5 and 17 weeks, respectively. As expected, the cage occupancies increase gradually on increasing the synthesis time. Note that, over a given period of time, the number of $\mathrm{CO}$ molecules in each $5^{12} 6^{2}$ cage is substantially smaller than that in $5^{12} 6^{4}$ cage.

Theoretical calculation. To understand the effects of cage occupancy on the stability of CO hydrates, we performed DFT calculations to determine the binding energies, $\Delta E$, of $\mathrm{CO}_{m^{-}}$ $\left(\mathrm{H}_{2} \mathrm{O}\right)_{n}$ clusters, which are calculated as

$$
\Delta E=E\left((\mathrm{CO})_{m}-\left(\mathrm{H}_{2} \mathrm{O}\right)_{n}\right)-E\left(\left(\mathrm{H}_{2} \mathrm{O}\right)_{n}\right)-m \times E(\mathrm{CO})
$$

where $m$ and $n$ are the numbers of enclosed $\mathrm{CO}$ and water molecules in the cage, respectively. The values of $\mathrm{n}$ corresponding to $5^{12}, 5^{12} 6^{2}$ and $5^{12} 6^{4}$ cages are, respectively, 20, 24 and 28 . Our optimized geometries for $\mathrm{H}_{2} \mathrm{O}$ cages are in good agreement with previous predictions by Patchkovskii et al. ${ }^{23}$ For the $\mathrm{CO}-\left(\mathrm{H}_{2} \mathrm{O}\right)_{20}$ cluster, the calculated binding energies are 3.47 and $2.59 \mathrm{kcal}$ $\mathrm{mol}^{-1}$ without and with zero point energy (ZPE) corrections, respectively. These values are comparable to the binding energy of the $\mathrm{CO}-\mathrm{H}_{2} \mathrm{O}$ complex ${ }^{24}$. The optimized structure shows that a $\mathrm{CO}$ molecule occupies the centre of each $5^{12}$ cage, with the $\mathrm{C}$ atom being $0.5 \AA$ from the cage centre. This is consistent with the MD simulation results using a classical force field, which will be discussed later. Instead of forming a single $\mathrm{CO}-\mathrm{H}_{2} \mathrm{O}$ complex, each $\mathrm{CO}$ molecule interacts with all surrounding $\mathrm{H}_{2} \mathrm{O}$ molecules.
The binding energies without and with ZPE correction for CO$\left(\mathrm{H}_{2} \mathrm{O}\right)_{24}\left(5^{12} 6^{2}\right.$ cage $)$ are 3.08 and $2.37 \mathrm{kcal} \mathrm{mol}^{-1}$, respectively, and those for $\mathrm{CO}-\left(\mathrm{H}_{2} \mathrm{O}\right)_{28}\left(5^{12} 6^{4}\right.$ cage $)$ are 2.76 and $2.14 \mathrm{kcal} \mathrm{mol}^{-1}$, respectively. Thus, water molecules in larger cages tend to have weaker binding (specifically van der Waals interaction) with $\mathrm{CO}$ molecules due to their larger separation. For the two CO molecules encapsulated in either a $5^{12} 6^{2}(\mathrm{sI})$ or $5^{12} 6^{4}$ (sII) cage, several trial positions, such as cage centre and cage edges facing the hexagonal and pentagonal rings, were also explored for possible $\mathrm{CO}$ occupancies. In both cases, no breakdown in cage symmetries was found, and the $\mathrm{CO}$ molecules eventually became equilibrated at the stabilized positions. However, the calculated binding energies for the optimized $(\mathrm{CO})_{2}-\left(\mathrm{H}_{2} \mathrm{O}\right)_{28}$ cluster without and with ZPE are 2.68 and $1.12 \mathrm{kcal} \mathrm{mol}^{-1}$, respectively, both of which are substantially lower than the sums of the corresponding binding energies for the two CO- $\left(\mathrm{H}_{2} \mathrm{O}\right)_{28}$ clusters. The binding energies of $(\mathrm{CO})_{2}-\left(\mathrm{H}_{2} \mathrm{O}\right)_{24}$ cluster with the ZPE correction are found to be very low or even negative, which is obviously due to the size effect of the sI large cage $\left(5^{12} 6^{2}\right)$. The occupation of two CO molecules in each $5^{12} 6^{2}$ cage can therefore significantly destabilize the sI CO clathrate structure.

\section{Discussion}

Figure 2 shows a schematic plot of the calculated binding energies for single and double occupancies of $\mathrm{CO}$ molecules in the large cages of sI and sII clathrates. Strikingly, there is a crossover in binding energy, indicating that beyond a critical point of cage occupancy the $5^{12} 6^{4}$ cages are energetically favoured over the $5^{12} 6^{2}$ cages in the clathrate structure. Also plotted in Fig. 2 are the cage occupancies determined from the neutron data shown in Fig. 1. The sI clathrate was initially observed after the synthesis time of 1 and 2.5 weeks because the $5^{12} 6^{2}$ cages are energetically favoured at small cage occupancies. However, when CO molecules are concentrated over a long period of time, the energetics is in favour of the $5^{12} 6^{4}$ cages enclosing two $\mathrm{CO}$

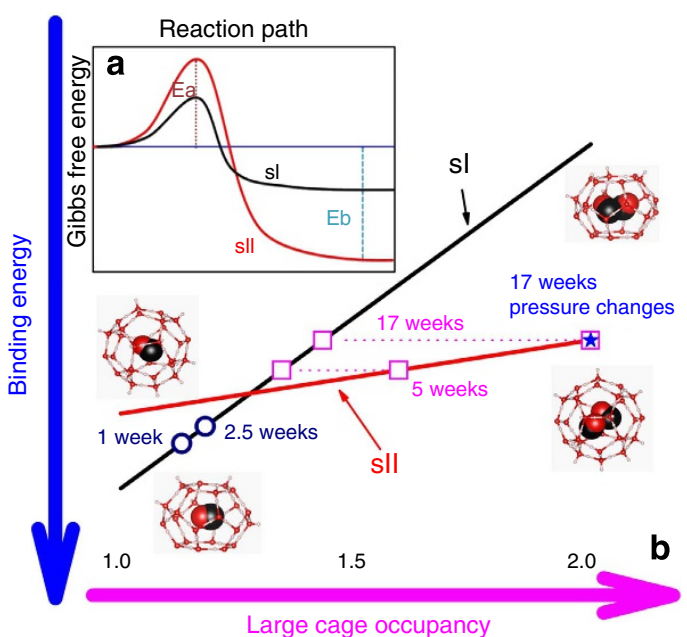

Figure 2 | Formation energetics of clathrate hydrates. (a) A schematic view of Gibbs free energy as a function of reaction coordinate for the formation of $\mathrm{sl}$ and sll carbon monoxide hydrates, inferred from our experimental observations (Fig. 1) and binding energy calculations. The horizontal line denotes the ground state of Gibbs free energy. $E_{\mathrm{a}}$ and $E_{\mathrm{b}}$ are activation energy and binding energy, respectively. The Gibbs free energy associated with $E_{\mathrm{b}}$ is negative as binding energy is released; (b) a schematic illustration of cage occupancy in $5^{12} 6^{2}$ and $5^{12} 6^{4}$ cages as a function of binding energy in $\mathrm{sl}$ and $\mathrm{sll}$ carbon monoxide clathrates at pressuretemperature conditions near the phase boundary. 
molecules and hence the sII hydrate. Our experiments show that relative to the sI phase, sII hydrate is thermodynamically favoured (lower Gibbs free energy) but not kinetically favoured (higher activation energy for the formation of stable, critical-sized hydrate nuclei). Therefore, at the final equilibrium state, sII hydrate must have higher binding energy than sI hydrate as illustrated in the inset of Fig. 2. This thermodynamic view is qualitatively consistent with our binding energy calculations. As also shown in Fig. 2, there is a region where sI and sII hydrates can coexist (marked by horizontal dot lines that intersect the two bold lines), which corresponds to the hydrates synthesized at 173 bar for 5 and 17 weeks. It should be pointed out that such coexistence can only be possible when $P-T$ conditions are in the vicinity of the sIsII hydrate phase boundary. As shown in the top pattern of Fig. 1, a phase-pure sII CO clathrate was obtained when the synthesis pressure was significantly lowered. On the other hand, an increase in pressure at a given temperature would favour the formation of sI hydrate ${ }^{20}$.

Our calculated binding energies for the optimized $(\mathrm{CO})_{2^{-}}$ $\left(\mathrm{H}_{2} \mathrm{O}\right)_{28}$ clusters are substantially lower than the sum of the binding energies for two individual $\mathrm{CO}-\left(\mathrm{H}_{2} \mathrm{O}\right)_{28}$ clusters. Therefore, the $(\mathrm{CO})_{2}-\left(\mathrm{H}_{2} \mathrm{O}\right)_{28}$ clusters in an isolated state are energetically unfavourable and may readily dissociate into $\mathrm{CO}-\left(\mathrm{H}_{2} \mathrm{O}\right)_{28}+\mathrm{CO}$. However, the interaction between $(\mathrm{CO})_{2}-\left(\mathrm{H}_{2} \mathrm{O}\right)_{28}$ clusters and their adjacent cages may play an important role in stabilizing the doubly occupied $5^{12} 6^{4}$ cages. The $5^{12} 6^{4}$ cages in sII clathrate, for example, are tightly connected to other $5^{12}$ cages and $5^{12} 6^{4}$ cages by sharing pentagonal faces and hexagonal faces. To gain insight into the distribution of $\mathrm{CO}$ molecules in hydrate cages and their intra- and inter-cage interactions, we performed a combined analysis of Rietveld refinement, MEM and MD simulations.
In sII CO hydrate, the $5^{12}$ cages share pentagons to form linear chains along the [110] direction, and each $5^{12} 6^{4}$ cage shares four hexagons with four neighbouring $5^{12} 6^{4}$ cages to form a diamond-shaped cage centre (Supplementary Fig. 1). The volume thermal expansion data were fitted to yield the parameters $\beta=8.2(17) \times 10^{-6} \mathrm{~K}^{-1}$ and $\gamma=8.7(2) \times 10^{-7} \mathrm{~K}^{-2}$ (Supplementary Fig. 2). Because CO molecule is smaller than the cage size of $5^{12}$ or $5^{12} 6^{4}$ and because the interaction between $\mathrm{CO}$ and the cages is via weak van der Waals force and even weaker dipole interactions, $\mathrm{CO}$ molecules in both cages have a disordered distribution. Figure 3 shows the distribution of $\mathrm{CO}$ molecules in $5^{12} 6^{4}$ and $5^{12}$ cages at $25 \mathrm{~K}$. CO molecules partially occupy small and large cages with the occupancy of $1 / 6$ (Table 1). Difference Fourier nuclear maps reveal that $\mathrm{CO}$ molecules are off-centred, as also demonstrated by the CO distribution from MD simulations in Fig. $3 b$ and nuclear density distribution from MEM in Fig. 3c. The off-centred character agrees well with the computed positions of the $\mathrm{CO}$ molecules in sI clathrate ${ }^{18,25}$. Note that, as a result of the disordered nature of guest molecules in the cages $^{22,26}$, the described CO distribution is only one of the most probable structural configurations from our Rietveld analyses, which, however, does not provide insight into the dynamics of guest molecules in the cages. On the other hand, a previous nuclear magnetic resonance (NMR) study on sI clathrate shows ${ }^{15,16}$ that the CO molecules in the large cages undergo anisotropic reorientation with substantial mobility even at $77 \mathrm{~K}$. The proposed model involves rapid motion of $\mathrm{CO}$ molecules among sites over each of the 14 faces, with the $\mathrm{CO}$ axis orientated towards the cage centre. Clearly, other techniques, such as NMR, Raman, infrared and inelastic scattering spectroscopies, are needed to provide more comprehensive
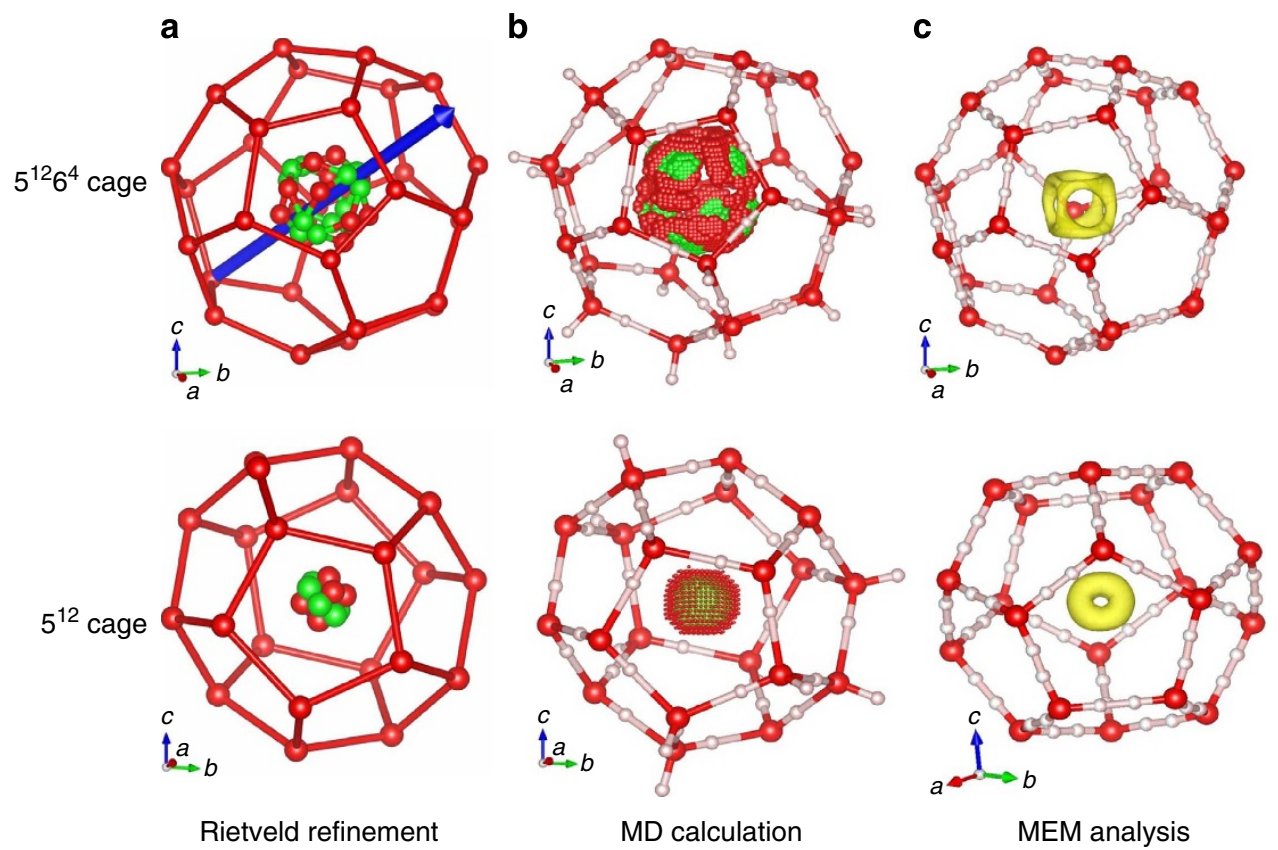

Figure 3 | Carbon monoxide distributions. The distribution of carbon monoxide molecules in $5^{12} 6^{4}$ and $5^{12}$ cages at $25 \mathrm{~K}$ (a) from Rietveld refinement; (b) from MD simulations with the $10 \%$ top possibilities of carbon monoxide molecular trajectories (oxygen in red and carbon in green) (Supplementary Fig. 3); and (c) from MEM analysis with isosurface level of $1.5 \mathrm{fm} \AA^{-3}$ for $5^{12} 6^{4}$ cage and $3 \mathrm{fm} \AA^{-3}$ for $5^{12}$ cage. The Pauling's half-hydrogen model with an average occupancy of 0.5 for each deuterium atom in water molecules was used for the Rietveld refinement and MEM analysis, and the ordered model was used for MD calculation. The green balls represent possible positions of carbon atoms, and the red balls represent oxygen atoms. In a $5^{12} 6^{4}$ cage, the 12 -fold coordinated carbon and oxygen positions are each related by a three-fold symmetry axis, and are normal to the hexagonal faces along the body diagonal of the cubic unit cell. The carbon atoms point towards the four hexagons and oxygen atoms point towards the four co-vertexes of the three pentagons. In a $5^{12}$ cage, the sixfold coordinated carbon and oxygen positions are each related by a three-fold body-diagonal axis, and the distribution of the carbon-oxygen bonds forms a solid angle of $102.6^{\circ}$. The carbon positions form a donut-shape ring in between the oxygen positions. 
Table 1 | Refined structural parameters of carbon monoxide sll clathrate at $25 \mathrm{~K}$.

Atom type

Schoenflies and international notations

Number of positions

Wyckoff notation

(a)

(e)

(g)

(e)

(g)

(g)

(g)

(g)

(i)

(g)

(g)

(g)

(g)
$D(5)$

$\mathrm{O}(\mathrm{S})^{\dagger}$

$C(S)^{\dagger}$

$\mathrm{O}(\mathrm{L})^{\star}$

$\mathrm{C}(\mathrm{L})^{\star}$
Coordinates of equivalent positions:

(000; $\left.01 \frac{1}{2} 1 / 2 ; 1 / 201 \frac{1}{2} ; 1 / 21 / 20\right)^{+}$

\section{Point of symmetry}

$T_{d}(-43 m)$

C $3 v(3 m)$

$\mathrm{CS}(\mathrm{m})$

C3v $(3 m)$

$\mathrm{C} 3 v(3 m)$

$\mathrm{CS}(m)$

$\mathrm{CS}(m)$

$\mathrm{CS}(m)$

$C 1(1)$

$\mathrm{CS}(m)$

$\operatorname{CS}(m)$

$\operatorname{CS}(m)$

$\operatorname{CS}(m)$

$\boldsymbol{x}$
$-1 / 8$
-0.217562
-0.182699
-0.157596
-0.185507
-0.195966
-0.203384
-0.142002
-0.088553
0.024985
-0.030239
0.304209
0.324843

y

$-1 / 8$

$-0.217562$

$-0.182699$

$-0.157596$

$-0.185507$

$-0.195966$

$-0.203384$

$-0.142002$

$-0.228918$

$-0.012822$

$-0.005751$

0.348103

0.401434
Occupancy

z

$-1 / 8 \quad 1.00$

$-0.217562$

1.00

$-0.369759$

$-0.157596$

$-0.185507$

$-0.315926$

$-0.272634$

$-0.371704$

0.146786

$-0.012822$

$-0.005751$

0.348103

0.324843
1.00

1.00

0.50

0.50

0.50

0.50

0.50

0.50

0.1667

0.1667

0.1667

0.1667

Details of site symmetries, coordinates and occupancies of oxygen, deuterium and carbon atoms in sll CO clathrate hydrate. While 16 small cages $\left(5^{12}\right)$ are singly occupied, 8 large cages $\left(5^{12} 6^{4}\right)$ are doubly occupied. For $\mathrm{CO}$ molecules in the two types of cages, we employed a disordered model in that the product of the cage occupancy and the number of Wyckoff positions divided by the cage numbers in a unit cell ( $165^{12}$ cages and $85^{12} 6^{4}$ cages) was equal to the number of CO molecules in each cage: one in $5^{12}$ and two in $5^{12} 6^{4}$. The data were collected at $25 \mathrm{~K}$ and analysed using the sll structure (space group $F d-3 m$ ), yielding $R w p=2.26 \%, R p=0.68 \%, R_{B}=4.83 \%$ and $a=17.0344(1) \AA$.

+ Denotes the CO molecules in large cages.

†Denotes the CO molecules in small cages.

understanding of the disordered state and dynamics of $\mathrm{CO}$ molecules in sII clathrate cages.

In the $5^{12} 6^{4}$ cages of sII clathrate, there are eight low-energy wells in two different groups favouring the occupancy of $\mathrm{CO}$ molecules: four in one group facing the four hexagonal rings of the $5^{12} 6^{4}$ cage, along the body diagonal of the unit cell, and the remaining four in the second group facing the co-vertex of three pentagonal rings of the $5^{12} 6^{4}$ cage, also along the body diagonal. Together, these eight positions form a cubic geometry with an edge length of $\sim 3 \AA$, which has the same orientation as the unit cell. In the $5^{12} 6^{4}$ cages, $\mathrm{CO}$ molecules are distributed at the eight vertices of the cube, which is different from the oblate shape of the CO distribution at the 14 sites towards each face of $5^{12} 6^{2}$ cage in sI clathrate ${ }^{15,16}$. Such distributions suggest that the intra-cage $\mathrm{CO}-\mathrm{H}_{2} \mathrm{O}$ interaction and inter-cage $\mathrm{CO}-\mathrm{CO}$ interaction can provide a significant source of stability for the sII framework. To corroborate this assumption, we have estimated the relative contribution of long-range interaction to the total stabilization of $\mathrm{CO}$ molecules in the large cage by calculating the trajectory average interaction energy of $\mathrm{CO}$ molecules within the $5^{12} 6^{4}$ cage. The stabilization energy of a CO molecule provided by the $5^{12} 6^{4}$ cage is determined to be $\sim 65 \%$ of the total interaction energy of the $\mathrm{CO}$ molecule, indicating that the molecules in the adjacent cages contribute $35 \%$ of the interaction energy for $\mathrm{CO}$ molecules in the doubly occupied cages. We have also found that the interaction between $\mathrm{CO}$ molecules inside a single cage has negative contribution to the stabilization of the system by energy decomposition analysis. Both of these findings are in good agreement with the results of our DFT calculations. It is worth pointing out that the structure and thermodynamic stability of clathrate hydrates are generally determined by two main factors: intermolecular interaction between guest and host molecules and the configurational entropy. The present work has focused on the former, but the latter contribution to the Gibbs free energy can also be important for guest molecules that strongly interact with the host framework water molecules. In addition, the stabilization mechanism by the adjacent cage interaction is yet to be fully understood, which represents another important area for future investigations. In $5^{12}$ cage, our MEM analysis demonstrates that CO molecules exhibit a doughnut-shaped density distribution, which is normal to the $\langle 111\rangle$ cubic diagonals (Fig. 3c).
Compared with the single occupancy in a $5^{12}$ cage, the enclosure of two CO molecules in a $5^{12} 6^{4}$ cage will increase the positional disorder and the scattering distributions (lower-symmetric position), which would in turn result in larger isotropic thermal motion $\left(U_{\text {iso }}\right)$ and displacement of $\mathrm{CO}$ from the cage centre.

Figure 4 shows the detailed CO distributions in sII cages obtained from MD simulation. As is shown, both $\mathrm{C}$ and $\mathrm{O}$ atoms in CO molecules are distributed off the centre of the $5^{12} 6^{4}$ cage. The $a-b$ plane projection of the density distributions displays four overlapped intensity maxima for the $\mathrm{CO}$ positions in the $5^{12} 6^{4}$ cage, indicating that the $C$ atoms are localized with the $\mathrm{O}$ atoms rotating around them, even though the $\mathrm{CO}$ molecules are allowed to move freely in the $5^{12} 6^{4}$ cage. Since such a configuration results from the combined effect of intercage $\mathrm{CO}-\mathrm{CO}$ and $\mathrm{CO}-\mathrm{H}_{2} \mathrm{O}$ interactions, the hollow shape of the $\mathrm{CO}$ distribution is expected to constitute a core that stabilizes both the $5^{12} 6^{4}$ cage of sII clathrate and the CO dimer contained within. Increasing the temperature would further delocalize the distribution of $\mathrm{CO}$ molecules in the cages, but they would still be confined within the potential energy surface well (Supplementary Fig. 4).

The radial distribution functions (RDFs) of $\mathrm{CO}$ molecules in sII clathrate from MD calculations are shown in Fig. 5. For the $5^{12}$ cage, the RDF is in excellent agreement with that reported for sI clathrate ${ }^{18,24}$. This is not unexpected given the similar cage geometries in sI and sII clathrates, even though the cages in the two structures have different symmetries. However, the RDF for $\mathrm{CO}$ molecules in the $5^{12} 6^{4}$ cage is significantly different from that in $\mathrm{sI}^{18}$. The peak values of both $\mathrm{C}$ and $\mathrm{O}$ atoms are lower for sII than for sI. There is no zero point at any distance as in the case of sI. This suggests that in the large cage of sII hydrate, CO molecules are more delocalized than those in sI and hence have a smoother potential energy surface. Thus, the larger cage size in sII not only increases the binding energy but also significantly lowers the potential barriers for $\mathrm{CO}$ molecules to move. The delocalization behaviour of $\mathrm{CO}$ is similar to that of $\mathrm{D}_{2}$ observed in hydrogen clathrate hydrate ${ }^{27}$, which also has a sII structure containing large $5^{12} 6^{4}$ cages. Thus, like all other hydrates of small guest molecules, the guest-framework interactions in $\mathrm{CO}$ clathrate hydrates may be dominated by the delocalization of encapsulated molecules inside their cages. 
a

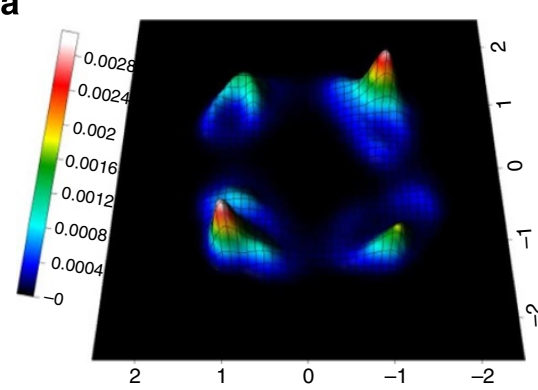

d

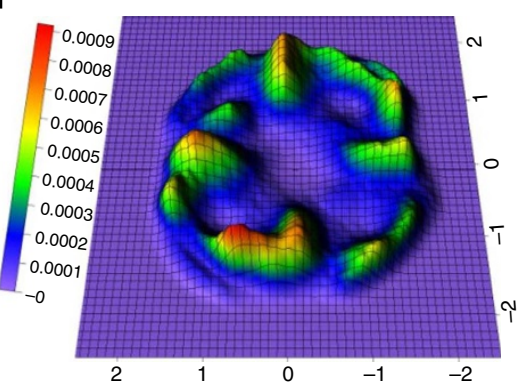

g

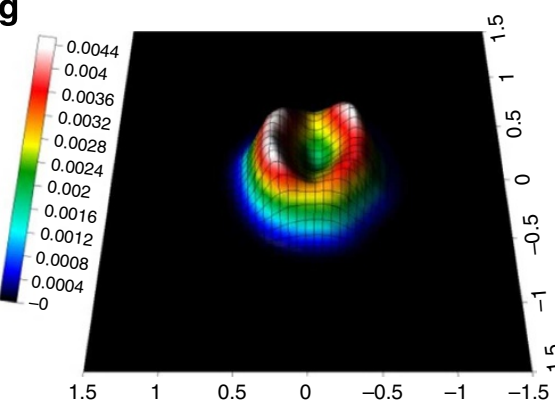

j

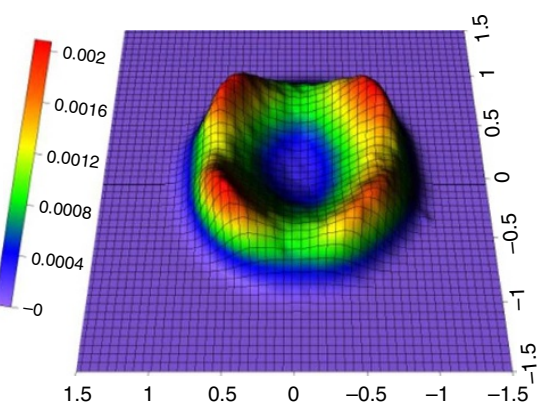

b

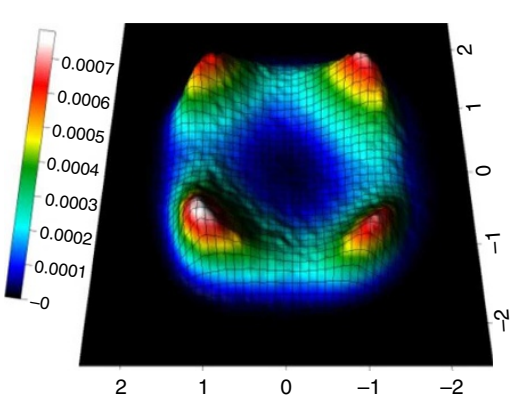

e

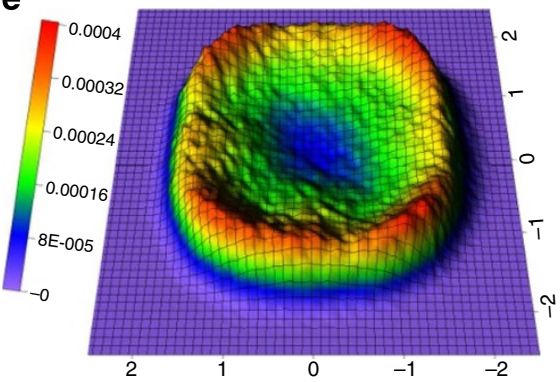

h

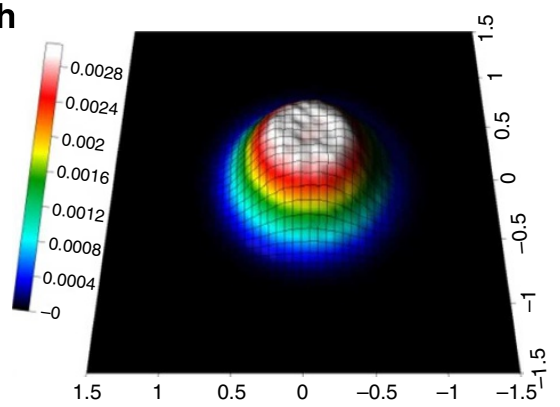

k

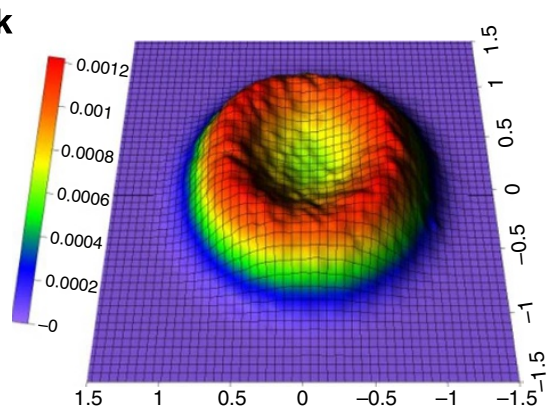

C

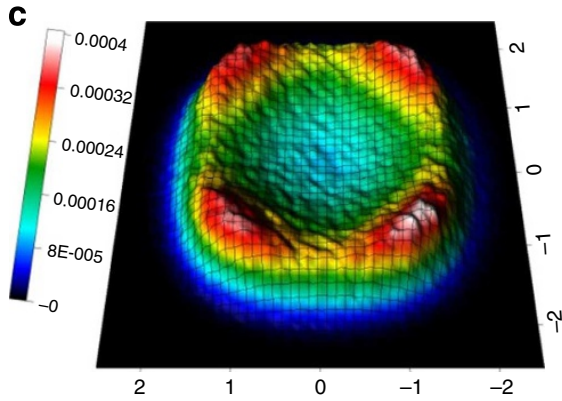

f

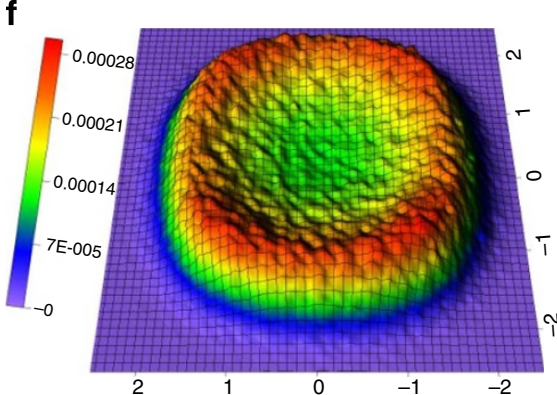

i

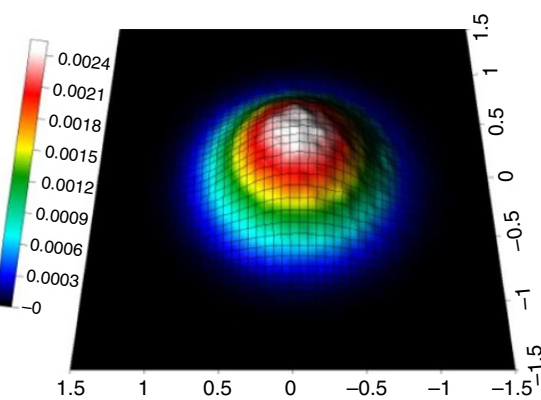

I

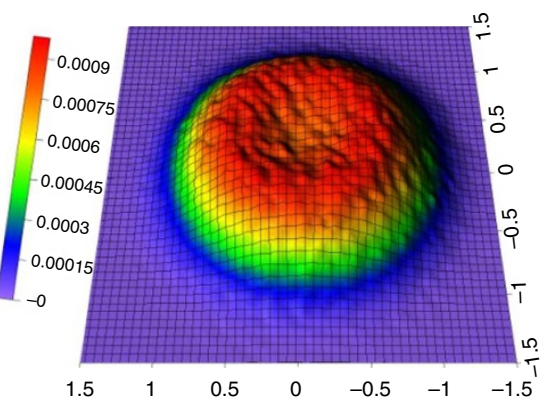

Figure 4 | Two-dimensional carbon monoxide distributions. The distribution of carbon monoxide molecules in sll clathrate from MD simulation:

(a-c) carbon atoms in carbon monoxide in $5^{12} 6^{4}$ cages at 25,100 and $260 \mathrm{~K}$, respectively; (d-f) oxygen atoms in carbon monoxide in $5^{12} 6^{4}$ cages at 25 , 100 and $260 \mathrm{~K}$, respectively; ( $\mathbf{g}-\mathbf{i})$ carbon atoms in carbon monoxide in $5^{12}$ cages at 25,100 and $260 \mathrm{~K}$, respectively; (j- $\left.\mathbf{I}\right)$ oxygen atoms in carbon monoxide in $5^{12}$ cages at 25,100 and $260 \mathrm{~K}$, respectively; the intensities are projected on the $a-b$ plane from around 10,000 carbon monoxide molecules. The cage centre is at coordinate $(0,0)$, and the axial unit is $\AA$. The intensity is the normalized density distribution of carbon monoxide in clathrate cages, and the summed total value is 1 in each cage.

In contrast to other clathrate hydrates, especially $\mathrm{N}_{2}$ and $\mathrm{O}_{2}$ hydrates, the structural behaviour of sI/sII CO hydrates has not been well established. For both sII $\mathrm{N}_{2}$ and $\mathrm{O}_{2}$ clathrates, the gas molecules in $5^{12} 6^{4}$ cages are doubly occupied, shifted off the cage centre and oriented along the diagonals of their cubic unit cells ${ }^{28,29}$, while in $5^{12}$ cages they are singly occupied at the cage centres and oriented along the $<100>$ axes. MD simulations of the dynamic behaviour of $\mathrm{N}_{2}$ and $\mathrm{O}_{2}$ in sII clathrates indicate that the molecular positions and orientations are essentially the same for $\mathrm{N}_{2}$ and $\mathrm{O}_{2}$ and are independent of the cage size and distortion $^{30}$. For comparison, the $\mathrm{CO}$ molecules in both $5^{12}$ and $5^{12} 6^{4}$ cages are off-centred. Notably, the CO molecules in $5^{12} 6^{4}$ cages rotate around the centre by forming a cubic symmetry, and in $5^{12}$ cages they are slightly off-centred with a pseudosphere configuration. In addition, an increased molecular freedom for $\mathrm{CO}$ molecules in large cages results in a distinct distribution of 

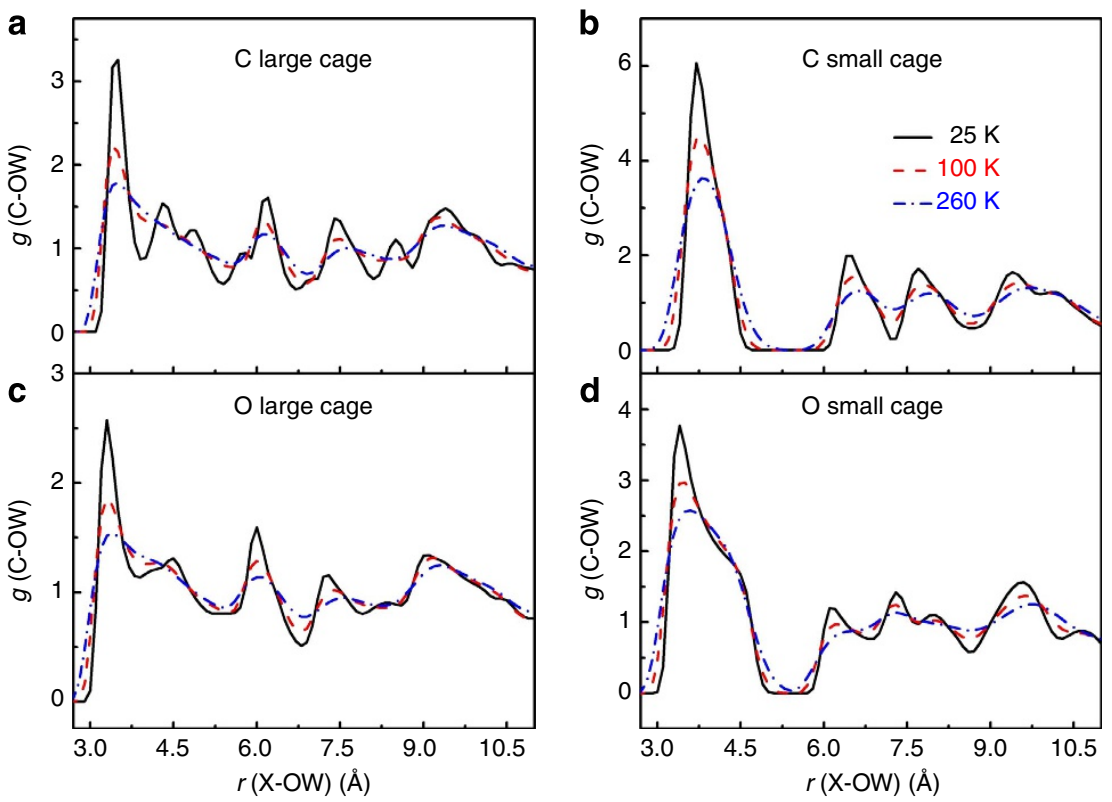

Figure 5 | Radial distribution function. Radial distribution functions of carbon atoms in carbon monoxide molecules in $5^{12} 6^{4}$ (a) and $5^{12}(\mathbf{b})$ cages, and oxygen atoms in carbon monoxide molecules in $5^{12} 6^{4}(\mathbf{c})$ and $5^{12}(\mathbf{d})$ cages at 25,100 and $260 \mathrm{~K}$ from MD simulation.

$\mathrm{CO}$ molecules with a hollow cubic geometry, whereas in small cages $\mathrm{CO}$ molecules are more confined/localized around the cage centre. With regard to the roles that dipole moment may play in the clathrate formation and stabilization, the present work does not provide a conclusive evidence. However, because $\mathrm{CO}$ has a small dipole moment, while $\mathrm{N}_{2}$ is non-dipole (note they are isoelectronic), the different formation sequences observed for $\mathrm{CO}$ hydrates may presumably arise from the $\mathrm{CO}$ dipole effect during the nucleation stage, although the difference in the guest chemistry can also be a determining factor. More specifically, the dipole moment could act as a turbulence factor through the interaction with the dipolar water molecule during the nucleation stage. This is a process that is difficult to address experimentally and warrants future kinetic MD simulations using high-performance computing. For instance, the kinetic MD simulations may be performed by starting with a mixture of $\mathrm{CO}$ gas + water and determining the role of the dipole moment in the polyhedral cage formation at early stages of hydrate nucleation. Together with NMR or Raman/IR spectroscopic experiments, the formation sequence of sI and sII CO clathrates and associated crystallization and growth kinetics can be established. It should be stressed that the present work is relevant only to the growth of $\mathrm{CO}$ hydrates after their nucleation. The early nucleation of clathrate hydrates, as demonstrated by molecular simulations, typically involves densification and high local concentration of guest molecules to form individual cages $^{31-33}$. These simulations also show that the clathrate nuclei are an amorphous mixture of polyhedral cages with structural motifs of a clathrate structure; hence, they do not have the same symmetry as the stable crystalline grains. In addition, the amorphous structure formed contains other types of cages that are not expected in an equilibrium hydrate structure. These characteristics of nucleation cannot be resolved using diffraction experiments.

In summary, based on the time-dependent study of the clathrate hydrate formation in the $\mathrm{CO}-\mathrm{H}_{2} \mathrm{O}$ system, we have demonstrated that sII hydrate can be formed in a time-evolving sequence after sI hydrate has initially crystallized. This finding validates previous hypotheses that sII CO hydrate would become more stable than sI CO hydrate when the concentration of $\mathrm{CO}$ molecules is saturated. This behaviour is associated with the difference in CO binding energy between $5^{12} 6^{2}$ and $5^{12} 6^{4}$ cages, where the $5^{12} 6^{4}$ cage in the sII structure is energetically favoured over the $5^{12} 6^{2}$ cage in the sI structure for double occupancy of $\mathrm{CO}$ molecules. More importantly, this is attributed to the crossover in the binding energy-cage occupancy space between the two cage types. As a result, a sII hydrate enclosing two $\mathrm{CO}$ molecules in $5^{12} 6^{4}$ cages can be stabilized at certain $P-T$ conditions through kinetically controlled cage filling. However, the $(\mathrm{CO})_{2}-\left(\mathrm{H}_{2} \mathrm{O}\right)_{28}$ clusters in an isolated state are energetically unfavourable and can readily dissociate into $\mathrm{CO}-\left(\mathrm{H}_{2} \mathrm{O}\right)_{28}$ and $\mathrm{CO}$. Our MD simulations suggest that the interactions between adjacent cages including $\mathrm{CO}$ $\mathrm{H}_{2} \mathrm{O}$ and CO-CO interactions provide a significant source of stability for the double-CO occupancy of hexakaidecahedral cage. Previous studies of $\mathrm{CO}$ hydrates indicate that both sI and sII CO hydrates may exist in the icy bodies of outer planets of the solar system, making them potentially important candidates for interpreting observed molecular anomalies ${ }^{12,13}$. Our comprehensive studies on their formation process, thermodynamic stability and encapsulation dynamics have provided valuable information for spectroscopic simulation and hence for detecting their occurrence in the solar system.

\section{Methods}

Neutron diffraction data collection. Time-of-flight neutron experiments were conducted at the HIPPO beamline, Los Alamos Neutron Science Center. The pressure cell was made of vanadium, which has a small coherent scattering for neutrons and can hold pressures up to 200 bar. To minimize the background of neutron patterns, deuterated water $\left(\mathrm{D}_{2} \mathrm{O}\right)$ instead of $\mathrm{H}_{2} \mathrm{O}$ was used for hydrate synthesis. Polycrystalline samples were prepared in a pressure vessel from ground $\mathrm{D}_{2} \mathrm{O}$ ice and gaseous $\mathrm{CO}$ at the initial pressure and temperature conditions ( 173 bar and $243 \mathrm{~K}$ ) used in ref. 15,16 As confirmed by our neutron diffraction measurements, the sI CO clathrate was initially formed during the first 1-4 weeks at 173 bar and $243 \mathrm{~K}$. A mixture of sI and sII CO clathrates was observed after 5 weeks, and, finally, a single-phase sII CO clathrate was obtained at $\sim 100$ bar and $252 \mathrm{~K}$ after 17 weeks (Fig. 1).

Rietveld refinement and MEM analysis. All neutron diffraction data were analysed using the GSAS package ${ }^{34}$, and the CO positions in the clathrate cages were determined from the difference Fourier nuclear maps and refined by 
subsequent Rietveld analyses. The initial atomic coordinates for the $\mathrm{D}_{2} \mathrm{O}$ framework were taken from a previous neutron diffraction study of $\mathrm{N}_{2}$ clathrate ${ }^{29}$ The $\mathrm{D}_{2} \mathrm{O}$ framework of sII CO clathrate and of the residual ice were both refined using Pauling's half-hydrogen model with an average occupancy of 0.5 for each deuterium atom ${ }^{35} \cdot \mathrm{D}_{2} \mathrm{O}$ in the framework can adopt six possible configurations as a result of the disordered orientation. However, diffraction experiments can only provide space- and time-averaged intra- and inter-molecular distances ${ }^{29}$, which may not reflect the instantaneous interactions between the framework cages and the guest molecules inside. The isotopic exchange between $\mathrm{D}$ in $\mathrm{D}_{2} \mathrm{O}$ ice and $\mathrm{H}$ atoms in atmospheric moisture was negligible and was ignored for all the refinements. To obtain the CO distributions in both small and large cages, difference nuclear Fourier maps were constructed after the best fit for the $\mathrm{D}_{2} \mathrm{O}$ framework was achieved. Our final Rietveld analysis revealed that in sII framework, each small cage is occupied by one $\mathrm{CO}$ molecule, whereas each large cage can be occupied by up to two $\mathrm{CO}$ molecules, resulting in a $\mathrm{CO} / \mathrm{D}_{2} \mathrm{O}$ ratio of $\sim 4 / 17$ when all the cages are fully occupied. The refined occupancies for $\mathrm{CO}$ molecules indicated one molecule in the $5^{12}$ cage and two in the $5^{12} 6^{4}$ cage; they were fixed in the subsequent analysis to better constrain the $\mathrm{CO}$ distributions in cages. In this study, the distribution of CO molecules in sII hydrate over a range of temperatures was analysed by the MEM/Rietveld analyses using data from four detector banks at $2 \theta=144.45^{\circ}, 119.89^{\circ}, 90.00^{\circ}$ and $39.30^{\circ}$. The corresponding $d$-spacings are in the range of $0.5-10.5 \AA$ (Supplementary Fig. 5). The observed structure factors, $F_{\mathrm{o}}$, and standard uncertainties, $\left(\left|F_{\mathrm{o}}\right|\right)$, were estimated with Alchemy ${ }^{36}$ from relevant data in files output by GSAS and analysed by MEM with Dysnomia ${ }^{37}$. The unit cell was divided into $128 \times 128 \times 128$ voxels. The detailed method for MEM analysis has been described in ref. 36 .

DFT and MD calculations. DFT was utilized in this work to provide further investigation on the stability of CO clathrate hydrates. The calculations utilized the Gaussian09 program package ${ }^{38}$. We optimized the structures of $\left(\mathrm{H}_{2} \mathrm{O}\right)_{20}\left(5^{12}\right.$ cage in both sI and sII), $\left(\mathrm{H}_{2} \mathrm{O}\right)_{28}\left(5^{12} 6^{4}\right.$ cage in sII) and $\left(\mathrm{H}_{2} \mathrm{O}\right)_{24}\left(5^{12} 6^{2}\right.$ cage in sI and their corresponding $\mathrm{CO}$ complexes, at the level of Becke 3-Parameter for exchange and Lee, Yang and Parr parameters for correlation). DFT (B3LYP)/6-31 + G(d) was used with the Coulomb-attenuating method ${ }^{39-41}$. All the stationary points were confirmed as minima by means of frequency calculations. To compare with our Rietveld refinement results of $\mathrm{CO}$ distributions in sII cages, we performed MD simulations with one CO molecule in each $5^{12}$ cage and two CO molecules in $5^{12} 6^{4}$ cage. A total of $\sim 100,000$ points from CO trajectories in $5 \mathrm{~ns}$ were projected on the $a-b$ plane, and the cage centres of both $5^{12}$ and $5^{12} 6^{4}$ cages were set as the zero points. The MD calculations were carried out at temperatures ranging from 25 to $260 \mathrm{~K}$ in canonical NVT (N-moles, V-volume, and T-temperature) ensembles obtained using the Nosé-Hoover thermostat ${ }^{42,43}$. The dimensions

of the simulation cells and their initial configurations were adopted using the experimental lattice parameters and geometries at corresponding temperatures. Three-dimensional periodic boundary conditions were applied. For the selection of proton configuration, the 'ice rules' (Bernal-Fowler ice rules) have been obeyed and the proton configuration with the minimum total dipole moment was selected. The TIP4P water model ${ }^{44}$ was used to calculate the interaction between $\mathrm{H}_{2} \mathrm{O}$ molecules. The interactions between $\mathrm{CO}$ and $\mathrm{H}_{2} \mathrm{O}$ molecules were modelled by the summation of electrostatic and Lennard-Jones-type interactions. The parameters were taken from Manesh et al. ${ }^{18}$, who used them to study the distribution of CO molecules in sI clathrate. The bond length of the CO molecule was fixed at the experimental value, $1.128 \AA$ (ref. 45). The Lennard-Jones parameters are $3.55 \AA$ and $0.3089 \mathrm{~kJ} \mathrm{~mol}^{-1}$ for $\mathrm{C}$ atoms and $2.95 \AA$ and $0.5120 \mathrm{~kJ} \mathrm{~mol}^{-1}$ for $\mathrm{O}$ atoms. The partial charges on the $\mathrm{C}$ and $\mathrm{O}$ atoms of the $\mathrm{CO}$ molecule were \pm 0.0223 in atomic units. The long-range electrostatic interactions were calculated with the ParticleParticle Particle-Mesh method ${ }^{46}$. The dimensions of the periodic simulation boxes were $34 \times 34 \times 34 \AA^{3}$, which were constructed by $2 \times 2 \times 2$ unit cells. Therefore, the simulation domain included a total of $1285^{12}$ cages and $645^{12} 6^{4}$ cages that incorporated a total of 1,088 water molecules. Each calculation was performed for 5.0 ns simulation time with a $1.0 \mathrm{fs}$ time step. The RDF and spatial density distribution of $\mathrm{CO}$ molecules respective to the centre of the corresponding cages were obtained from MD trajectories. All MD simulations in this work were performed using the LAMMPS program suite ${ }^{47}$.

\section{References}

1. Fortes, A. D. \& Choukroun, M. Phase behaviour of ices and hydrates. Space Sci. Rev. 153, 185-218 (2010).

2. Khokhar, A. A., Gudmundsson, J. S. \& Sloan, E. D. Gas storage in structure H hydrates. Fluid Phase Equilibr. 150-151, 383-392 (1998).

3. Ripmeester, J. A. \& Ratcliffe, C. I. ${ }^{129}$ Xe NMR studies of clathrate hydrates: new guests for structure II and structure H. J. Phys. Chem. 94, 8773-8776 (1990).

4. Schicks, J. M. \& Ripmeester, J. A. The coexistence of two different methane hydrate phases under moderate pressure and temperature conditions: kinetic versus thermodynamic products. Angew. Chem. Int. Ed. 43, 3310-3313 (2004).

5. Loveday, J. S., Nelmes, R. J., Klug, D. D., Tse, J. S. \& Desgreniers, S. Structural systematics in the clathrate hydrates under pressure. Can. J. Phys. 81, 539-544 (2003).
6. Loveday, J. S., Nelmes, R. J. \& Guthrie, M. High-pressure transitions in methane hydrate. Chem. Phys. Lett. 350, 459-465 (2001).

7. Loveday, J. S. et al. Stable methane hydrate above $2 \mathrm{GPa}$ and the source of Titan's atmospheric methane. Nature 410, 661-663 (2001).

8. Staykova, D. K., Kuhs, W. F., Salamatin, A. N. \& Hansen, T. Formation of porous gas hydrates from ice powders: diffraction experiments and multistage model. J. Phys. Chem. B 107, 10299-10311 (2003).

9. Kuhs, W. F., Chazallon, B., Radaelli, P. G. \& Pauer, F. Cage occupancy and compressibility of deuterated N2-clathrate hydrate by neutron diffraction. J. Incl. Phenom. Mol. Recog. Chem. 29, 65-77 (1997).

10. Yang, L. et al. Synthesis and characterization of a new structure of gas hydrate. Proc. Natl Acad. Sci. USA 106, 6060-6064 (2009).

11. Lewis, J. S. \& Prinn, R. G. Kinetic inhibition of $\mathrm{CO}$ and $\mathrm{N}_{2}$ reduction in the solar nebula. Astrophys. J. 238, 357-364 (1980).

12. Lunine, J. I. \& Stevenson, D. J. Thermodynamics of clathrate hydrate at low and high pressure with application to the outer solar system. Astrophys. J. Suppl. Ser. 58, 493-531 (1985).

13. Miller, S. L. in Ices in the Solar System. (eds Klinger, J., Benest, D., Dollfus, A. \& Smoluchowski, R.) Vol. 59 (Reidel, 1985).

14. Sloan, E. D. Fundamental principles and applications of natural gas hydrates. Nature 426, 353-359 (2003).

15. Davidson, D. W. et al. A clathrate hydrate of carbon monoxide. Nature 328, 418-419 (1987).

16. Desando, M. A., Handa, Y. P., Hawkins, R. E., Ratcliffe, C. I. \& Ripmeester, J. A. Dielectric and ${ }^{13} \mathrm{C}$ NMR studies of the carbon monoxide clathrate hydrate. J. Incl. Phenom. Mol. Recog. Chem. 8, 3-16 (1990).

17. Mohammadi, A. H., Anderson, R. \& Tohidi, B. Carbon monoxide clathrate hydrates: equilibrium data and thermodynamic modeling. AIChE J. 51, 2825-2833 (2005).

18. Mohammadi-Manesh, H., Alavi, S., Woo, T. K., Ashrafizaadeh, M. \& Najafi, B. Molecular dynamics simulation of ${ }^{13} \mathrm{C}$ NMR powder lineshapes of $\mathrm{CO}$ in structure I clathrate hydrate. Phys. Chem. Chem. Phys. 11, 8821-8828 (2009).

19. Mohammadi, A. H. \& Richon, D. Ice - clathrate hydrate - gas phase equilibria for air, oxygen, nitrogen, carbon monoxide, methane, or ethane + water system. Ind. Eng. Chem. Res. 49, 3976-3979 (2010).

20. Dartois, E. C. O. Clathrate hydrate: near to mid-IR spectroscopic signatures. Icarus 212, 950-956 (2011).

21. Ballard, A. L. \& Sloan, E. D. The next generation of hydrate prediction: I. Hydrate standard states and incorporation of spectroscopy. Fluid Phase Equilibr. 194, 371-383 (2002).

22. Alavi, S., Udachin, K. \& Ripmeester, J. A. Effect of guest-host hydrogen bonding on the structures and properties of clathrate hydrates. Chem. Eur. J. 16, 1017-1025 (2010).

23. Patchkovskii, S. \& Tse, J. S. Thermodynamic stability of hydrogen clathrates. Proc. Natl Acad. Sci. USA 100, 14645-14650 (2003).

24. Lundell, J. \& Latajka, Z. Vibrational calculations for the $\mathrm{H}_{2} \mathrm{O}$... CO complex. J Mol. Struct. 887, 172-179 (2008)

25. Cwiklik, L. \& Devlin, J. P. Hindering of rotational motion of guest molecules in the type I clathrate hydrate. Chem. Phys. Lett. 494, 206-212 (2010).

26. Takeya, S., Udachin, K. A., Moudrakovski, I. L., Susilo, R. \& Ripmeester, J. A. Direct space methods for X-ray powder diffraction for guest-host materials: applications to cage occupancies and guest distributions in clathrate hydrates. $J$. Am. Chem. Soc. 132, 524-531 (2010).

27. Alavi, S., Ripmeester, J. A. \& Klug, D. D. Molecular-dynamics study of structure II hydrogen clathrates. J. Chem. Phys. 123, 024507-024513 (2005).

28. Van Klaveren, E. P., Michels, J. P. J., Schouten, J. A., Klug, D. D. \& Tse, J. S. Stability of doubly occupied $\mathrm{N}_{2}$ clathrate hydrates investigated by molecular dynamics simulations. J. Chem. Phys. 114, 5745-5754 (2001).

29. Chazallon, B. \& Kuhs, W. F. In situ structural properties of $\mathrm{N}_{2}-, \mathrm{O}_{2}-$, and air-clathrates by neutron diffraction. J. Chem. Phys. 117, 308-320 (2002).

30. Horikawa, S., Itoh, H., Tabata, J., Kawamura, K. \& Hondoh, T. Dynamic behavior of diatomic guest molecules in clathrate hydrate structure II. J. Phys. Chem. B 101, 6290-6292 (1997).

31. Walsh, M. R., Koh, C. A., Sloan, E. D., Sum, A. K. \& Wu, D. T. Microsecond simulations of spontaneous methane hydrate nucleation and growth. Science 326, 1095-1098 (2009).

32. Walsh, M. R. et al. The cage, dynamics, and structuring of incipient methane clathrate hydrates. Phys. Chem. Chem. Phys. 13, 19951-19959 (2011).

33. Jacobson, L. C., Hujo, W. \& Molinero, V. Amorphous precursors in the nucleation of clathrate hydrates. J. Am. Chem. Soc. 132, 11806-11811 (2010).

34. Larson, A. C. \& Von Dreele, R. B. General Structure Analysis System (GSAS) 86-748 (Los Alamos National Laboratory Report LAUR, 2004).

35. Pauling, L. The structure and entropy of ice and of other crystals with some randomness of atomic arrangement. J. Am. Chem. Soc. 57, 2680-2684 (1935).

36. Izumi, F. \& Kawamura, Y. Three-dimensional visualization of nuclear densities by MEM analysis from time-of-flight neutron powder diffraction data. Bunseki Kagaku 55, 391-395 (2006). 
37. Izumi, F. \& Momma, K. Three-dimensional visualization of electron- and nuclear-density distributions in inorganic materials by MEM-based technology. IOP Conf. Ser.: Mater. Sci. Eng. 18, 022001 (2011).

38. Gaussian 09, Revision A.1Frisch, M. J. et al. Gaussian, Inc., Wallingford, CT USA (2009).

39. Becke, A. D. Density-functional thermochemistry. III. The role of exact exchange. J. Chem. Phys. 98, 5648-5652 (1993).

40. Lee, C., Yang, W. \& Parr, R. G. Development of the Colle-Salvetti correlationenergy formula into a functional of the electron density. Phys. Rev. B 37, 785-789 (1988).

41. Yanai, T., Tew, D. P. \& Handy, N. C. A new hybrid exchange-correlation functional using the Coulomb-attenuating method (CAM-B3LYP). Chem. Phys. Lett. 393, 51-57 (2004).

42. Evans, D. J. \& Holian, B. L. The Nose-Hoover thermostat. J. Chem. Phys. 83, 4069-4074 (1985)

43. Martyna, G. J., Klein, M. L. \& Tuckerman, M. Nosé-Hoover chains: the canonical ensemble via continuous dynamics. J. Chem. Phys. 97, 2635-2643 (1992).

44. Horn, H. W. et al. Development of an improved four-site water model for biomolecular simulations: TIP4P-Ew. J. Chem. Phys. 120, 9665-9678 (2004).

45. Gilliam, O. R., Johnson, C. M. \& Gordy, W. Microwave spectroscopy in the region from two to three millimeters. Phys. Rev. 78, 140-144 (1950).

46. Sadus, R. J. in Molecular Simulation of Fluids 162-169 (Elsevier Science, 1999).

47. Plimpton, S. Fast parallel algorithms for short range molecular dynamics.

J. Comp. Phys. 117, 1-19 (1995).

\section{Acknowledgements}

This work was supported by the laboratory-directed research and development (LDRD) program of Los Alamos National Laboratory, which is operated by Los Alamos National Security LLC under DOE Contract No. DE-AC52-06NA25396. The experimental work has benefited from the use of the Lujan Neutron Scattering Center at Los Alamos

Neutron Science Center, which is funded by the US Department of Energy's Office of
Basic Energy Sciences. The work at Institute of Physics, Chinese Academy of Sciences (IOPCAS) was funded by CAS project under contract Nos: KJCX2-YW-W26 and XDB07000000. We thank the High Pressure Science and Engineering Center (HiPSEC), UNLV for support. This research was sponsored in part by the National Nuclear Security Administration under the Stewardship Science Academic Alliances program through DOE Cooperative Agreement \#DE-NA0001982.

\section{Author contributions}

J.L.Z, X.Y., H.X., Y.Z. and C.J. conceived the work; J.L.Z. and X.Y. conducted the experiments with the help of S.C.V and C.J.; S.D., T.C.G. and J.S.F. conducted the MD simulation; J.L.Z., Y.K., F.I. and K.M. did the MEM analysis; J.L.Z analyzed the data and drafted the manuscript; J.Z.Z., H.X., C.J., and Y.Z. helped edit the manuscript and also provided input for the Discussion section.

\section{Additional information}

Supplementary Information accompanies this paper at http://www.nature.com/ naturecommunications

Competing financial interests: The authors declare no competing financial interests.

Reprints and permission information is available online at http://npg.nature.com/ reprintsandpermissions/

How to cite this article: Zhu, J. et al. Encapsulation kinetics and dynamics of carbon monoxide in clathrate hydrate. Nat. Commun. 5:4128 doi: 10.1038/ncomms5128 (2014)

This work is licensed under a Creative Commons AttributionNonCommercial-ShareAlike 4.0 International License. The images or other third party material in this article are included in the article's Creative Commons license, unless indicated otherwise in the credit line; if the material is not included under the Creative Commons license, users will need to obtain permission from the license holder to reproduce the material. To view a copy of this license, visit http:// creativecommons.org/licenses/by-nc-sa/4.0/ 